\title{
Taiwanese Depression Questionnaire and AD8 Questionnaire for Screening Late-Life Depression in Communities
}

This article was published in the following Dove Press journal: Neuropsychiatric Disease and Treatment

\author{
Yi-Chia Wei iD $^{1-4}$ \\ Li-Yuan Huang ${ }^{1,2,5}$ \\ Chemin Lin $\mathbb{1}^{1,2,6}$ \\ Yu-Chiau Shyu (D) 1,7 \\ Chih-Ken Chen ${ }^{1,2,6}$
}

'Community Medicine Research Center, Chang Gung Memorial Hospital, Keelung, Taiwan; ${ }^{2}$ College of Medicine, Chang

Gung University, Taoyuan, Taiwan;

${ }^{3}$ Department of Neurology, Chang Gung

Memorial Hospital, Keelung, Taiwan;

${ }^{4}$ Institute of Neuroscience, National Yang-Ming University, Taipei, Taiwan;

${ }^{5}$ Department of Nursing, Chang Gung Memorial Hospital, Keelung, Taiwan;

${ }^{6}$ Department of Psychiatry, Chang Gung Memorial Hospital, Keelung, Taiwan;

${ }^{7}$ Department of Nursing, Chang Gung

University of Science and Technology,

Taoyuan, Taiwan
Correspondence: Chih-Ken Chen

Department of Psychiatry, Chang Gung

Memorial Hospital at Keelung, No. 200

Lane 208, Jijin Ist Road, Anle District,

Keelung City, 204, Taiwan

Tel +886-2-24329292-2725

Fax+886-2-243I593।

Email kenchen@cgmh.org.tw
Background: Major depressive disorder (MDD) presents with emotional and somatic symptoms and sometimes subjective cognitive complaints (SCCs). This study developed a collaborative method to integrate SCC assessment for evaluating late-life MDD.

Methods: Residents aged $>50$ years in the Community Medicine Research Center of Keelung Chang Gung Memorial Hospital in Taiwan during 2017-2018 were prospectively recruited in this study. The participants were asked to report their depressive tendency and SCCs using the Taiwanese Depression Questionnaire (TDQ) and the $\mathrm{AD} 8$, respectively, and were administered psychiatric evaluation through the MiniInternational Neuropsychiatric Interview (MINI). The participants were divided into elderly (age $\geq 65$ years) and older adult (age 50-65) groups. The MDD predictive powers were assessed using logistic regression and receiver operating characteristic (ROC) curve analyses.

Results: Of the 118 enrolled participants (mean age: 64.81 \pm 4.99 , female-to-male ratio: 1.62), 9, 21, and 88 were categorized as those with current MDD, past MDD, and nonMDD on the basis of the MINI results, respectively. After adjustments for age, sex, and sleep quality, the TDQ score (odds ratio: 1.152, $\mathrm{p}=0.003$ ) and AD8 score (odds ratio: 1.710, $\mathrm{p}=0.020$ ) were used individually to predict current MDD. Overall, the TDQ individually predicted current MDD well with area under the ROC curve (AUC) of 0.835 ( $p=0.001$ ). However, in the elderly group ( $\mathrm{N}=63)$, the TDQ score did not identify current MDD well (AUC: $0.780, \mathrm{p}=0.063$ ). After co-considering SCCs, the linear combination of the sum of the TDQ score and four folds of the AD8 score could effectively distinguished elderly people with current MDD from those without it (AUC: 0.875, $\mathrm{p}=0.013$ )-with the cutoff of the aforementioned combined score being $\geq 32$.

Conclusion: The self-reported response to the TDQ is a feasible approach of identifying MDD in community-dwelling people. Combining TDQ and AD8 scores further improved depression detection in elderly people.

Keywords: geriatric depression, major depressive disorder, Taiwanese Depression Questionnaires, subjective cognitive complaint, AD8

\section{Background}

Major depressive disorder (MDD) is an important disease with wide distribution across age groups and the world, with substantial severity that can threaten individuals' socioeconomic behavior. MDD prevalence is $16.2 \%$ overall and $6.6 \%$ annually. ${ }^{1}$ Effectively detecting depression has become a crucial goal in public mental health. However, emotional expression patterns vary on the basis of the 
culture. ${ }^{2}$ The Taiwanese Depression Questionnaire (TDQ) is a culturally relevant questionnaire used in epidemiological surveys in Taiwan. ${ }^{3}$ In Taiwan, the TDQ has been used to screen depression in patients with chronic pain, ${ }^{4}$ patients with head and neck cancers, ${ }^{5}$ and hospital physicians. $^{6}$

Depression in elderly is becoming prevalent and thus is an important health issue. ${ }^{7}$ Geriatric depression could present as multidomain involvements, including apathy, poor appetite, chronic illness with body pain, sleep disturbance, or mimic of cognitive impairment with impaired episodic memory or slow psychomotor speed. ${ }^{8,9}$ Therefore, the detection and assessment of geriatric depression could be confusing because of the miscellaneous clinical variability. Data suggest significant differences in the form and quality of clinical presentation of depression between younger and older people, and therefore, the atypical presentations of depression in elderly people typically cannot be completely assessed using conventional measures. ${ }^{10,11}$

Psychomotor tardiness and passive refusal to appropriately respond to cognitive tests are typical symptoms of "pseudodementia," which is depression mistaken for dementia. ${ }^{12,13}$ Cognitive complaints and depressive presentations had reciprocal effects and were frequently accompanied by anxiety and stress. ${ }^{14}$ Of the various factors mediating cognitive symptoms in depression, such as the duration of the current depression episode and presence of disability, age is a crucial factor. ${ }^{15}$ Depression presents more memory impairment, poorer verbal learning, and slower motor speed in older individuals than in younger individuals. ${ }^{16}$ Notably, atypical presentations of depression in elderly people were considered while developing the Geriatric Depression Scale (GDS). In 1982, Yesavage noted that psychomotor retardation and passive refusal lead to misdiagnosis of depression as dementia. ${ }^{17}$ Because cognitive complaints in elderly individuals are generally high, identifying late-life depression is challenging. Although comprehensive cognitive assessments help to identify late-life depression, a well-trained evaluator is necessary to conduct these assessments, which limits their use. ${ }^{18}$

Several self-report questionnaires have been developed to identify geriatric depression. The GDS, one of the recommended self-report scales, ${ }^{11}$ consists of questions pertaining to subjective memory concerns ("Do you feel you have more problem with memory than most?") and comparisons of current cognitive performances with those of the past ("Is your mind as clear as it used to be?").
Although the GDS is well validated and is being used in different countries, ${ }^{19-21}$ the translated Taiwanese version of the GDS encountered cultural differences and could not effectively distinguish depressed and nondepressed populations. ${ }^{22}$ Therefore, a new convenient self-rating scale for weighing subjective cognitive complaints (SCCs) that can be modified according to cultural peculiarities and idiomatic vocabulary is mandatory for evaluating late-life depression. This study tested the validity of the TDQ for screening depression in older Taiwanese people who live in Northern Taiwan and assessed the effectiveness of incorporating self-reported cognitive decline in predicting depression.

\section{Methods}

From December 2017 to December 2018, volunteers aged $\geq 50$ years were recruited from the Community Medicine Research Center of Keelung Chang Gung Memorial Hospital, Taiwan. The participant age range was based on the typical age of the occurrence of increased SCCs. ${ }^{23}$ These community-dwelling participants completed the TDQ for depression screening and Ascertain Dementia 8-Item Informant questionnaire (AD8) for reporting SCCs. Well-trained researchers assisted the participants while filling out the questionnaires if they had difficulties reading or answering.

The TDQ had 18 culturally relevant depression screening questions and yielded a total score ranging from 0 to 40. The items in the TDQ included questions regarding somatic symptoms of depression and emotional destress, which were crying, depression, agitation, insomnia, anorexia, overeating, chest tightness, uneasiness, weakness, libido loss, annoyance, poor memory, poor concentration, retardedness, poor self-confidence, pessimism, suicidal tendency, guilty feeling, anhedonia, and general discomfort. The original cutoff TDQ score was 19 , with $\geq 19$ points indicating depression and $<19$ points indicating no depression. $^{3}$

The AD8 is a screening tool for cognitive impairment, which is composed of eight questions, with one point for each question. ${ }^{23}$ The items in the AD8 are regarding the cognitive performance of common people in daily life, which were judgment, interest, repetition, application, orientation, finance, appointments, and consistency. ${ }^{24}$ An AD8 score $\geq 2$ suggests cognitive impairment. The AD8 can be informantbased $^{25}$ or self-reported. ${ }^{26}$ In this study, participants were asked to compare their current daily cognitive functions with those 5 years ago and report their subjective cognitive 
impairment. The AD8 used in this study was the traditional Chinese version, which was well validated in Taiwan people. $^{23}$

A formal psychiatric interview of participants was conducted by using the Mini-International Neuropsychiatric Interview (MINI), which is a well-validated structured interview for diagnosing psychiatric disorders. ${ }^{27,28}$ Moreover, the participants were evaluated for cognitive abilities, sleeping quality, and quality of life by using the Mini-Mental State Examination (MMSE), ${ }^{29}$ Pittsburgh Sleep Quality Index (PSQI), ${ }^{30}$ and Short Form (36-item) Health Survey (SF-36), respectively. ${ }^{31}$

On the basis of MINI results, participants were divided into current MDD, past MDD, and non-MDD groups. The groups were compared with respect to basic demographic data of age, sex, school education year, marital status, and annual income. Furthermore, the groups were compared for TDQ score, AD8 score, MMSE score, and suicide risk. Chi square test and Student's $t$-test were used to evaluate differences in nominal and continuous data, respectively.

Correlations between the TDQ and AD8, MMSE, PSQI, and SF-36 scores were analyzed using Spearman's rho statistics. A logistic regression model was used to evaluate the odds ratio (OR) of MDD diagnosis by using the MINI. Various combinations of variables were used in the regression model, including the TDQ score only, the AD8 score only, a combination of the TDQ score, AD8 score, age (whether $>65$ years), and sex, and a combination of the TDQ score, AD8 score, age, sex, PSQI score, and MMSE score. The receiver operating characteristic (ROC) curve was used to depict the TDQpredicted MDD in comparison with that diagnosed using MINI. Furthermore, the predictive value of a linear combination of TDQ and AD8 scores was tested using the area under the ROC curve (AUC). An AUC $>0.5$ indicated that target values could be effectively classified. Owing to the possibility of atypical presentations of MDD, such as SCCs in elderly people, and an increase in SCCs with age, enrollees were separated into elderly (age $\geq 65$ years) and older adult (age 50-65 years) groups to examine whether age influenced the likelihood of major depression. In the prediction model, the cutoff score was determined based on the highest Youden's J statistics $[($ sensitivity + specificity $)-1]$ in the model. A p value of $<0.05$ was considered statistically significant. Statistical analysis was performed using IBM SPSS Statistics (version 20).

\section{Results}

Of the 127 community-dwelling residents from the northern coastal area of Taiwan, 9 were excluded because of incomplete data in the TDQ, AD8, or MINI. Finally, 118 residents were enrolled, with a mean age of $64.81 \pm 4.99$ years and a female-to-male ratio of 1.62. The participants were divided into groups of current $\operatorname{MDD}(\mathrm{N}=9)$, past $\operatorname{MDD}(\mathrm{N}=21)$, and non-MDD $(\mathrm{N}=88)$. The betweengroup comparisons showed higher proportions of women (88\%, 76.2\%, and 55.7\%, respectively; $\mathrm{p}<0.05)$, widows $(33.3 \%, 20.0 \%$, and $8.4 \%$, respectively; $\mathrm{p}<0.01)$, and divorcees $(22.2 \%, 20.0 \%$, and $3.6 \%$, respectively; $\mathrm{p}=$ 0.003 ) in the current MDD and past MDD groups than in the non-MDD group (Table 1). Both TDQ scores $(25.56 \pm$ $10.90,17.57 \pm 11.56$, and 9.49 $\pm 8.70 ; \mathrm{p}<0.001)$ and AD8 scores $(4.00 \pm 2.45,2.43 \pm 1.96$, and $1.83 \pm 2.12 ; \mathrm{p}=$ 0.012 ) were higher in the current MDD and past MDD groups than in the non-MDD group. A high percentage risk of suicide was identified in people with current MDD $(55.6 \%)$. Furthermore, PSQI scores revealed that the current and past MDD groups had poorer sleep quality than did the non-MDD group $(9.56 \pm 4.10,10.06 \pm 4.31$, and $7.15 \pm 3.80 ; \mathrm{p}=0.012$ ). No difference was found in age, years of education, income level, or cognition status in terms of MMSE score (Table 1).

Of the 118 enrolled participants, 63 and 55 belonged to elderly and older adult groups, respectively. Comparisons between elderly and older adult groups revealed differences only in MMSE scores $(27.56 \pm 2.31$ and $28.72 \pm$ 1.33, $\mathrm{p}=0.001)$. The demographics, depressive scores, and SCC scores were not considerably different between the groups (Table S1).

Spearman's rho statistics were used to evaluate correlations among age; years of education; income level; as well as TDQ, AD8, MMSE, PSQI, and SF-36 scores. Statistics revealed a weak correlation between TDQ and AD8 scores, with a correlation coefficient of 0.386 at a significance of $\mathrm{p}<0.01$ (Table S2). Depressive tendency slightly increased with SCCs. Moreover, the depressive score on the TDQ was moderately correlated with sleep quality in terms of the PSQI (correlation coefficient 0.561, $\mathrm{p}<0.001)$ and quality of life with respect to general health (correlation coefficient $-0.564, \mathrm{p}<0.001$ ), vitality (correlation coefficient $-0.688, \mathrm{p}<0.001)$, and mental health (correlation coefficient $-0.603, \mathrm{p}<0.001$ ) per the SF-36 questionnaire. These results suggest that sleeping quality and quality of life deteriorate as depressive traits increase. 
Table I Comparison of Baseline and Scoring According to the MINI Diagnosis of MDD

\begin{tabular}{|c|c|c|c|c|}
\hline & Current MDD (N=9) & Past MDD (N=2I) & Non-MDD $(\mathbf{N}=\mathbf{8 8})$ & $p$ \\
\hline Age & $63.22 \pm 2.68$ & $65.14 \pm 3.86$ & $64.90 \pm 5.40$ & 0.601 \\
\hline Sex (female) & $8(88.9 \%)$ & $16(76.2 \%)$ & 49 (55.7\%) & $0.049 *$ \\
\hline Years of school education & $7.78 \pm 3.63$ & $11.65 \pm 3.68$ & $9.77 \pm 4.40$ & 0.060 \\
\hline $\begin{array}{c}\text { Marital status: } \\
\text { Married } \\
\text { Divorced } \\
\text { Widowed }\end{array}$ & $\begin{array}{l}4(44.4 \%) \\
2(22.2 \%) \\
3(33.3 \%)\end{array}$ & $\begin{array}{l}12(60.0 \%) \\
4(20.0 \%) \\
4(20.0 \%)\end{array}$ & $\begin{array}{c}73(88.0 \%) \\
3(3.6 \%) \\
7(8.4 \%)\end{array}$ & $0.003 *$ \\
\hline $\begin{array}{l}\text { Annual income level }\left(10^{3} \text { USD): }\right. \\
\quad<10 \\
\quad 10-30 \\
30-60 \\
>60 \\
\text { Concealed }\end{array}$ & $\begin{array}{l}3(33.3 \%) \\
3(33.3 \%) \\
\text { I (II.I\%) } \\
\text { I (II.I\%) } \\
\text { I (II.I\%) }\end{array}$ & $\begin{array}{c}3(15.0 \%) \\
10(50.0 \%) \\
6(30.0 \%) \\
0(0.0 \%) \\
1(5.0 \%)\end{array}$ & $\begin{array}{c}26(32.9 \%) \\
29(36.7 \%) \\
16(20.3 \%) \\
4(5.1 \%) \\
4(5.1 \%)\end{array}$ & 0.638 \\
\hline $\begin{array}{l}\text { TDQ score: } \\
\text { TDQ total } \\
\text { TDQ } \geq 19\end{array}$ & $\begin{array}{c}25.56 \pm 10.90 \\
8(88.9 \%)\end{array}$ & $\begin{array}{c}17.57 \pm 11.56 \\
\mid \mathrm{I}(52.4 \%)\end{array}$ & $\begin{array}{c}9.49 \pm 8.70 \\
13(14.8 \%)\end{array}$ & $\begin{array}{l}<0.001^{*} \\
<0.001^{*}\end{array}$ \\
\hline $\begin{array}{c}\text { MINI-suicide: } \\
\text { High risk } \\
\text { Low risk } \\
\text { No risk }\end{array}$ & $\begin{array}{l}5(55.6 \%) \\
3(33.3 \%) \\
I(11.1 \%)\end{array}$ & $\begin{array}{c}0(0.0 \%) \\
5(23.8 \%) \\
16(76.2 \%)\end{array}$ & $\begin{array}{c}0(0.0 \%) \\
2(2.3 \%) \\
86(97.7 \%)\end{array}$ & $<0.001^{*}$ \\
\hline $\begin{array}{l}\text { AD8 score: } \\
\text { AD8 total } \\
\text { AD8 } \geq 2\end{array}$ & $\begin{array}{c}4.00 \pm 2.45 \\
7(77.8 \%)\end{array}$ & $\begin{array}{c}2.43 \pm 1.96 \\
13(61.9 \%)\end{array}$ & $\begin{array}{l}1.83 \pm 2.12 \\
42(47.7 \%)\end{array}$ & $\begin{array}{l}0.012 * \\
0.146\end{array}$ \\
\hline MMSE score & $27.56 \pm 1.59$ & $28.70 \pm 1.03$ & $27.98 \pm 2.21$ & 0.259 \\
\hline PSQI score & $9.56 \pm 4.10$ & $\mid 0.06 \pm 4.31$ & $7.15 \pm 3.80$ & $0.012 *$ \\
\hline
\end{tabular}

Notes: *Statistic significant at 0.05 level. The between group comparison was analyzed by one-way ANOVA. The post hoc 2 -sided Dunnett $t$ test used non-MDD group as control group. TDQ score was significantly higher in both current MDD and past MDD groups when compared to non-MDD group ( $p=0.00 \mathrm{I}, 95 \% \mathrm{Cl} 2.90-13.26 ; p<0.00 \mathrm{I}$, $95 \% \mathrm{Cl} 8.6 \mathrm{I}-23.53)$. AD8 score was significantly higher in current MDD group than non-MDD group $(p=0.008,95 \% \mathrm{Cl} 0.49-3.85)$, but was not significantly different between past MDD and non-MDD groups $(p=0.429)$.

Abbreviations: MINI, Mini-International Neuropsychiatric Interview; MDD, major depressive disorder; USD, United States Dollar; TDQ, Taiwanese depression questionnaire; MMSE, Mini-Mental State Examination; PSQI, Pittsburgh Sleep Quality Index.

In the logistic regression model for the MINI-based MDD diagnosis, demographic variables and variables having a significant correlation with depressive traits were tested, which included age, sex, and scores of TDQ, AD8, and PSQI. TDQ and AD8 scores individually predict current MDD by $1.127(\mathrm{p}=0.001)$ and $1.463(\mathrm{p}=0.012)$ folds of odds, respectively (Table $2 \mathrm{~A}$, models 1 and 2). Old age (age $\geq 65$ ), sex, sleep quality, and TDQ and AD8 scores are significant predictors of current MDD (OR: $1.152, \mathrm{p}=0.003$; OR: $1.710, \mathrm{p}=0.020$; Table $2 \mathrm{~A}$, model 3). In regression models for both current and past MDD, TDQ and AD8 scores individually provided the diagnosis (Table 2B, models 1 and 2). However, the
AD8 was not included when considering other variables (Table 2B, model 3).

The ROC curve was used to evaluate self-reported TDQ and AD8 performance in MDD diagnosis. A linear combination of the TDQ and AD8 was created from their approximate ratio of $B$ values in regression models (Table 2) as the sum of the TDQ score and four folds of the AD8 score. Table 3 and Figure 1 summarize the performances of the TDQ, AD8, and linear combination of TDQ and AD8 in ROC curves to predict either current MDD or current and past MDD in all participants, the elderly group, or older adult group. In general, the TDQ alone predicted current MDD well, and adding 
Table 2 Logistic Regression Model to Determine MINI Diagnosis of MDD

\begin{tabular}{|c|c|c|c|c|c|c|c|c|c|c|c|c|}
\hline & \multicolumn{4}{|c|}{ Model I } & \multicolumn{4}{|c|}{ Model 2} & \multicolumn{4}{|c|}{ Model 3} \\
\hline & B & SE & $p$ & OR & B & $S E$ & $p$ & OR & B & $S E$ & $p$ & $O R$ \\
\hline TDQ & 0.119 & 0.036 & $0.001 *$ & 1.127 & & & & & 0.141 & 0.047 & $0.003 *$ & 1.152 \\
\hline AD8 & & & & & $0.38 I$ & 0.151 & $0.012 *$ & 1.463 & 0.536 & 0.231 & $0.020 *$ & 1.710 \\
\hline Sex & & & & & & & & & 2.457 & 1.413 & 0.082 & 11.668 \\
\hline Age $\geq 65$ & & & & & & & & & 0.468 & 0.920 & 0.611 & 1.597 \\
\hline PSQI & & & & & & & & & -0.078 & 0.120 & 0.517 & 0.925 \\
\hline
\end{tabular}

(B) Binary logistic regression models for current and past MDD

\begin{tabular}{|c|c|c|c|c|c|c|c|c|c|c|c|c|}
\hline & \multicolumn{4}{|c|}{ Model I } & \multicolumn{4}{|c|}{ Model 2} & \multicolumn{4}{|c|}{ Model 3} \\
\hline & B & $S E$ & $p$ & OR & B & SE & $p$ & OR & B & $S E$ & $p$ & OR \\
\hline TDQ & 0.096 & 0.023 & $<0.001 *$ & 1.101 & & & & & 0.100 & 0.031 & $0.001 *$ & I.105 \\
\hline AD8 & & & & & 0.217 & 0.095 & $0.023 *$ & 1.242 & 0.083 & 0.131 & 0.527 & 1.086 \\
\hline Sex & & & & & & & & & 1.562 & 0.681 & $0.022 *$ & 4.769 \\
\hline Age $\geq 65$ & & & & & & & & & -0.369 & 0.566 & 0.514 & 0.691 \\
\hline PSQI & & & & & & & & & 0.050 & 0.077 & 0.510 & 1.052 \\
\hline
\end{tabular}

Note: *Statistic significant at 0.05 level.

Abbreviations: MINI, Mini-International Neuropsychiatric Interview; MDD, major depressive disorder; TDQ, Taiwanese depression questionnaire; PSQI, Pittsburgh Sleep Quality Index; SE, standard error; OR, odds ratio.

the AD8 score did not significantly improve the model (AUC $=0.835$ for the TDQ score $[p=0.001], 0.751$ for the AD8 score [p $=0.013]$, and 0.887 for the TDQ-AD8 combination $[p<0.001]$; Table 3 ). In the elderly group, prediction based on the TDQ or AD8 score alone was unsatisfactory, yielding insignificant AUCs of $0.780(\mathrm{p}=$ $0.063)$ and $0.627(\mathrm{p}=0.398)$, respectively. However, the TDQ-AD8 combination offered much improved predictive performance, with an AUC of $0.875(p=0.013)$. In older adult group, both the TDQ and AD8 were individually good predictors of current MDD (AUC of $0.892[\mathrm{p}$ $=0.004]$ and $0.804[\mathrm{p}=0.026]$, respectively). The TDQ-
AD8 combination was an even stronger predictor, with the AUC value of $0.918(\mathrm{p}=0.002)$. Calculating Youden's J statistics as [(sensitivity + specificity $)-1]$ yielded the best cutoffs of TDQ for predicting MDD, which were identical to those originally proposed for clinical use: a TDQ score of 19 with a sensitivity, specificity, positive predictive value, and negative predictive value of $0.889,0.780,88.9 \%$ and $77.3 \%$, respectively. The TDQ-AD8 combination score of $\geq 31$ feasibly predicted current MDD diagnosis, with a sensitivity, specificity, positive predictive value, and negative predictive value of $1.000,0.789,100 \%$, and $77.1 \%$, respectively.

Table 3 AUC of the ROC Curves

\begin{tabular}{|l|l|l|l|l|}
\hline $\begin{array}{l}\text { Classification AUC } \\
(p, \mathbf{9 5} \% \mathbf{C l})\end{array}$ & Variable & All Subjects $\mathbf{( N = I ~ 1 8 )}$ & Elderly $(\mathbf{N}=63)$ & Older Adults $(\mathbf{N}=55)$ \\
\hline Current MDD & TDQ & $0.835(0.001,0.694-0.976) *$ & $0.780(0.063,0.496-1.000)$ & $0.892(0.004,0.789-0.995) *$ \\
& AD8 & $0.751(0.013,0.606-0.895) *$ & $0.627(0.398,0.385-0.869)$ & $0.804(0.026,0.684-0.924) *$ \\
& TDQ+4*AD8 & $0.887(<0.001,0.818-0.956) *$ & $0.875(0.013,0.791-0.959) *$ & $0.918(0.002,0.818-1.000) *$ \\
\hline Current and past MDD & TDQ & $0.752(<0.001,0.636-0.867) *$ & $0.623(0.162,0.431-0.815)$ & $0.879(<0.001,0.763-0.995) *$ \\
& AD8 & $0.655(0.011,0.548-0.763) *$ & $0.558(0.514,0.390-0.725)$ & $0.709(0.016,0.57 I-0.847) *$ \\
& TDQ+4*AD8 & $0.755(<0.001,0.646-0.864) *$ & $0.649(0.092,0.457-0.840)$ & $0.848(<0.001,0.740-0.956) *$ \\
\hline
\end{tabular}

Notes: TDQ+4*AD8 was the linear combination of the approximate ratio of the $B$ values of TDQ and AD8 in the regression model (Table 2) as the sum of TDQ score and 4 folds of AD8 score. *Statistically significant at $p<0.05$.

Abbreviations: AUC, area under curve; ROC, receiver operating characteristic; MDD, major depressive disorder; TDQ, Taiwanese depression questionnaire; Cl, confidence interval. 
A

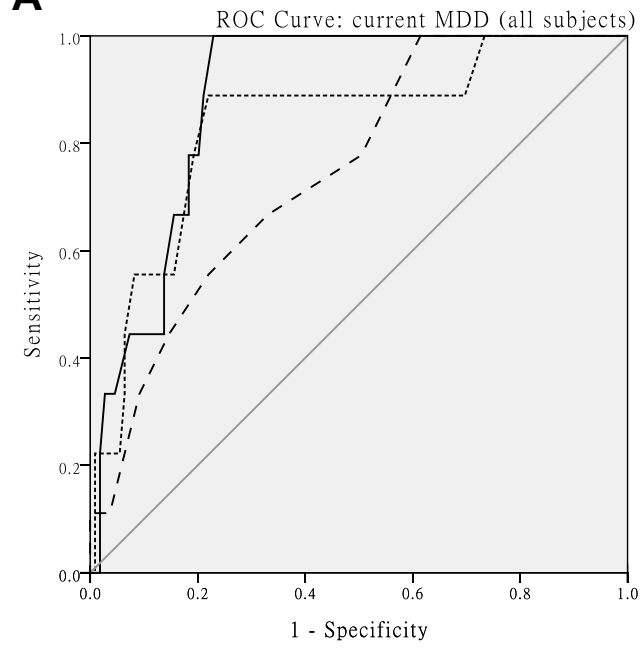

C

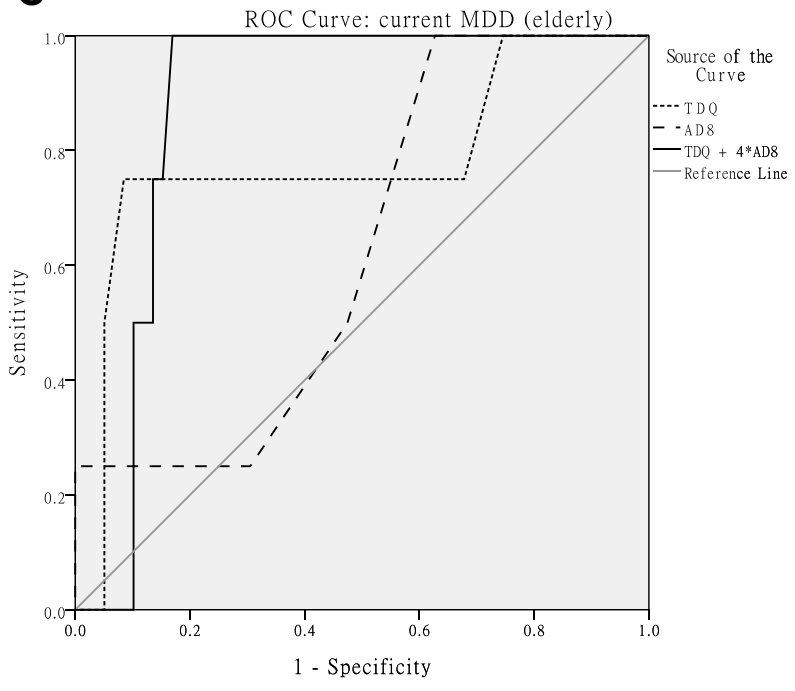

$\mathbf{E}$

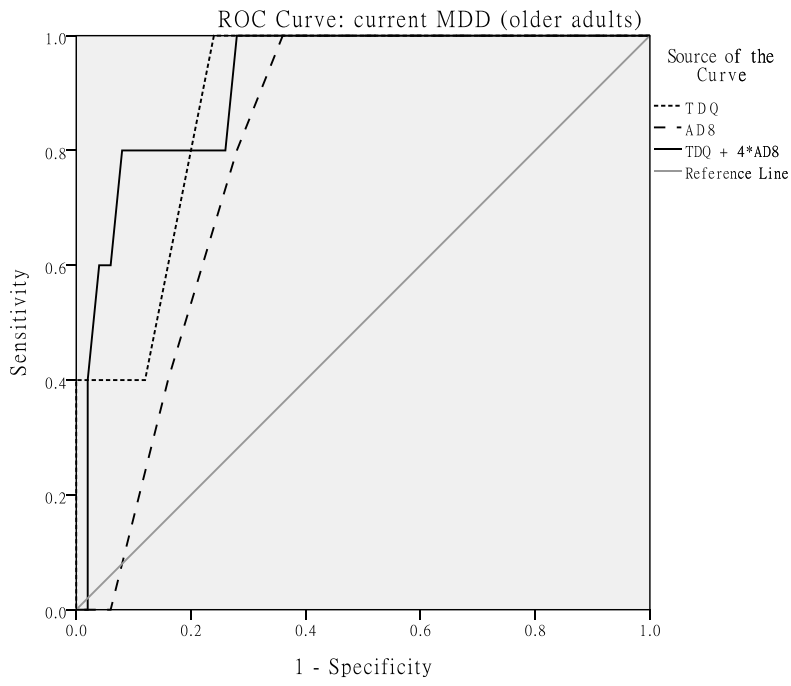
Source of the
Curve
-- TDQ
- - AD 8
- TDQ + 4*AD8
- Reference Line
B

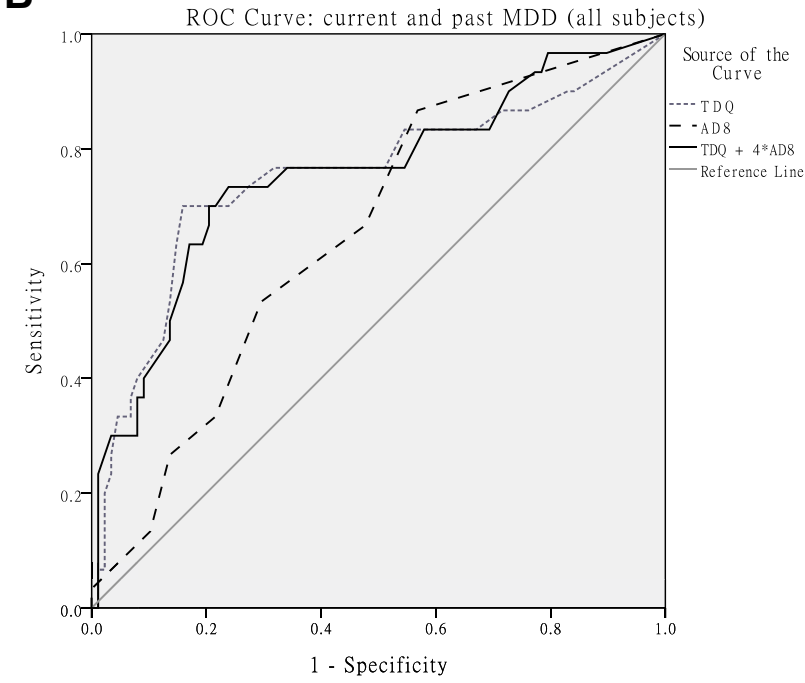

D

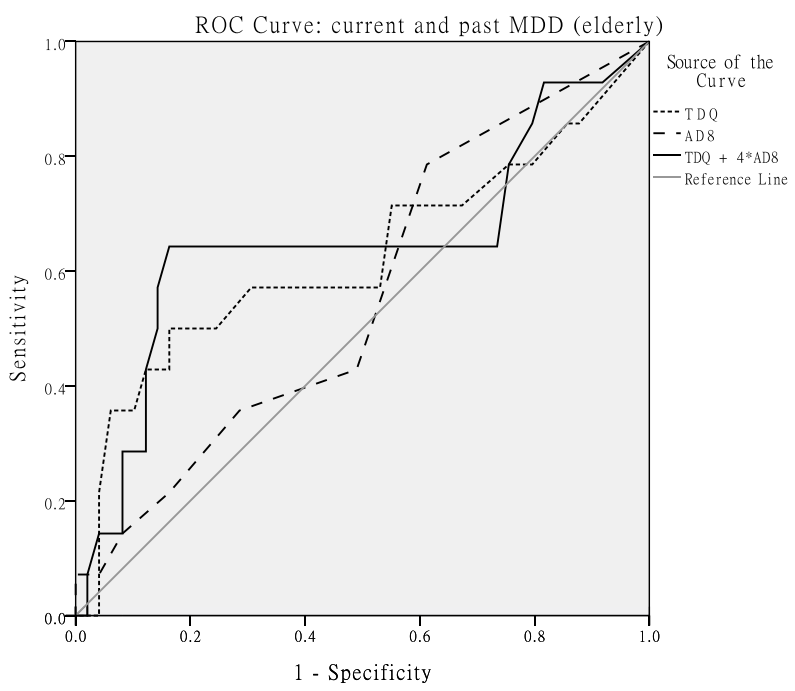

$\mathbf{F}$

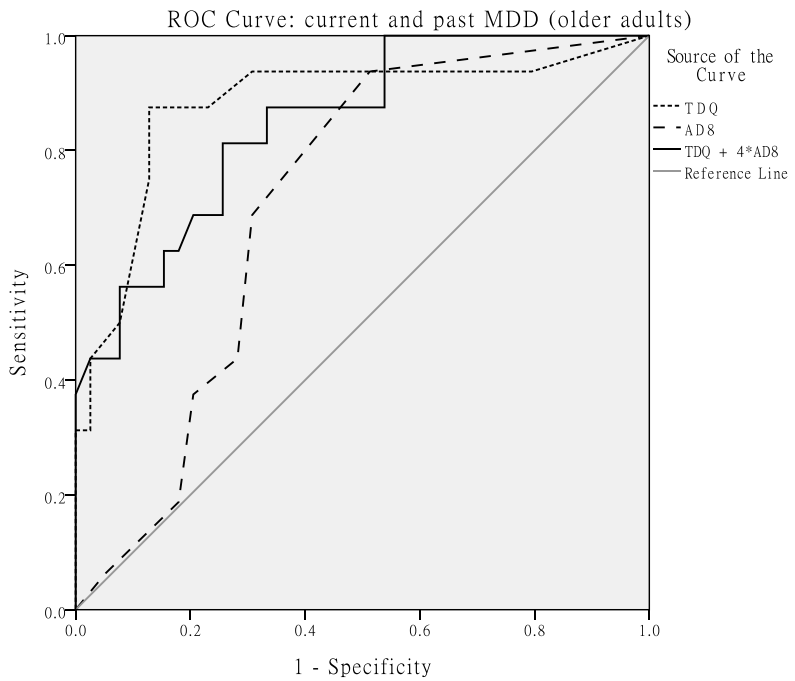

Figure I ROC curves of identifying participants with depressive disorders. ROC curves of all subjects (A, B), the elderly group (C, D) and the older adult group (E, F). The targets of the ROC curves were either current MDD (A, C, E) or both current and past MDD (B, D, F). The AUC of the curves were listed in Table 3. 
The Youden's statistics in the elderly group and older adult group revealed similar cutoffs at $\geq 32$ and $\geq 31$, respectively (Figure $\mathrm{S} 1$ ).

Regarding ROC curves for both current and past MDD, the general performance of these three predictors was less favorable than their performance in predicting current MDD. In the elderly group, neither the TDQ or AD8 score individually nor the TDQ-AD8 combination reached statistical significance. In the older adult group, the TDQ alone could predict current and past MDD well (AUC 0.879, $\mathrm{p}<0.001$; Table 3 and Figure 1).

\section{Discussion}

This cross-sectional investigation of the cohort study was conducted at the Community Medicine Research Center of Keelung Chang Gung Memorial Hospital in Northern Taiwan. Using self-reported responses to questionnaires on depression and SCCs, this work aimed to screen people with major depression. Group analysis, correlation analysis, logistic regression modeling, and ROC curve analysis on 118 community residents confirmed that the TDQ score can be useful in distinguishing people with and without MDD. A new score equal to a linear combination of TDQ and AD8 scores outperformed the TDQ score alone in identifying current MDD in elderly people.

Depression is a multifactorial disease associated with socioeconomic function, culture, and interpersonal relationships. Comparisons of patients with current MDD, past MDD, and non-MDD demonstrated that sex ratio and marital status percentages differed among these groups (Table 1). A previous review concluded that women are more likely to encounter depressive disorder. ${ }^{32}$ Marital disruption, including divorce and widowhood, precipitate depression, ${ }^{33,34}$ and this fact may explain the high percentage of nonmarried people with current MDD. Other objective criteria such as demographic characteristics, education, and financial status did not lead to major depression.

Depression, a complicated mental illness, manifests as multidomain symptoms. Using more than one tool to coevaluate depression is feasible to improve detection accuracy. Because depression is commonly concomitant with cognitive complaints, as determined in our earlier study of subjective cognitive decline, ${ }^{26}$ we combined tools focused on depression and SCCs for detecting depression. The TDQ is a self-reporting depressive questionnaire on somatic and emotional aspects. The AD8 is a selfreporting questionnaire that covers various cognitive domains in daily life. Correlation tests in Table S2 revealed a weak correlation between TDQ and AD8 scores. Therefore, depression complaints and SCCs should be considered together, and collinearity was not a problem in the regression model. Under these assumptions, TDQ and AD8 scores together predicted current MDD in the logistic regression (Table $2 \mathrm{~A}$, model 3) and improved predictive power through their linear combination (Figure 1 and Table 3). Therefore, co-considering depressive and cognitive complaints is a feasible means of improving major depression diagnosis. Similar attempts of combining measurements of SCCs and other scales were made in detecting dementia. ${ }^{35}$ Furthermore, machine learning models to predict depression was beneficial when multiple questionnaires were integrated. In these studies, each questionnaire had certain features to help identify depression. $^{36,37}$ This is the additive value of using multidomain assessments in the mental illnesses having multidomain manifestations.

Early-life and late-life depression have their unique features. The original goal of creating the TDQ was to overcome cultural differences by using certain terms and expressing symptoms in certain ways pertaining to specific cultures, but it did not consider the effect of age on depression presentations. ${ }^{3}$ Nevertheless, the simple question used in the TDQ is suitable to inquire elder people. By contrast, the AD8 was originally created for detecting SCCs of older adults and elderly. ${ }^{23,25}$ In this work, integrating subjective cognitive survey with a traditional depression survey provided additive value in identifying depression in elderly people aged $>65$ years. Neither the TDQ nor the AD8 score alone could identify depression in elderly people, but a linear combination of AD8 and TDQ scores as a new predictor significantly improved the ROC curve (Figure 1C and Table 3). However, additional time and attention needed to answer two questionnaires might be a burden for elderly people. The total number of questions of the TDQ-AD8 combination was 26-more than the 15 questions in the simplified GDS (GDS-15) but less than the 30 questions in the full version of the GSD (GDS30). A meta-analysis that compared these two versions of the GDS recommended using GDS-15 over GDS-30 in primary care. ${ }^{38}$ In this case, a higher number of questions did not reach better diagnostic validity. However, the extra-short version of the GDS with four or five questions did not gain better validity than that of GDS-15. ${ }^{39}$ Therefore, a proper length and composition of questionnaire is crucial for identifying depression in elderly 
people. In this study, participants comfortably answered the 26 questions. A standby assistant might also have helped. Based its time efficiency and diagnostic benefit, the use of TDQ-AD8 combination is encouraged.

This study has several limitations. First, this study had a relatively small number of people with current major depression, which might restrict result generalization. Second, the study recruited community-dwelling people, and their visits to the hospital for complete psychiatric evaluation required their cooperation. Those willing to undergo that evaluation might have been more active and have had a greater ability to move than the others. People with major depression lack motivation and therefore may have refused to participate. Consequently, the number of people with MDD could be under-estimated. Third, grouping the enrolled people based on age group to generate ROC curves further reduced the statistical power. To overcome these limitations, increasing the case number and random sampling of community population will improve the statistic power. Finally, this study did not include a comparative tool focused particularly on geriatric depression, such as the GDS, and therefore, improvement in the detection rate of late-life depression with the TDQAD8 combination could not be objectively weighed. In future, a validation study to compare the GDS and TDQAD8 combination could overcome this limitation.

\section{Conclusions}

The TDQ can feasibly be used for screening major depression in community-dwelling people. Incorporating SCCs by using the AD8 further improved the identification of depression, particularly current MDD in elderly people.

\section{Abbreviations}

MDD, major depressive disorder; TDQ, Taiwanese depression questionnaire; MINI, Mini-International Neuropsychiatric Interview; MMSE, Mini-Mental State Examination; PSQI, Pittsburgh Sleep Quality Index; SF-36, Short Form (36-item) Health Survey; ROC, receiver operating characteristic; AUC, area under the curve.

\section{Data Sharing Statement}

The datasets used and/or analyzed during the current study are available from the corresponding author on reasonable request.

\section{Ethics Approval and Consent to Participate}

This study was approved by the Institutional Review Board of Chang Gung Memorial Hospital, under approval no. 201600270B0 and no. 201600580B0. All the participants had been well informed about the study and had signed written consents before entering the study. This study was conducted in accordance with the Declaration of Helsinki.

\section{Acknowledgments}

All authors thank the Community Medicine Research Center, Chang Gung Memorial Hospital, Keelung for resource supports.

\section{Author Contributions}

All authors contributed to data analysis, drafting or revising the article, have agreed on the journal to which the article will be submitted, gave final approval of the version to be published, and agree to be accountable for all aspects of the work.

\section{Funding}

This study was fund by Chang Memorial Hospital Research Project CRRPG2G0051, CRRPG2G0052, CRRPG2G0071, CRRPG2G0072, CLRPG2H0041 and CLRPG2L0051 to support designing the study and collecting of data.

\section{Disclosure}

The authors declare that they have no competing interests.

\section{References}

1. Kessler RC, Berglund P, Demler O, et al. The epidemiology of major depressive disorder: results from the National Comorbidity Survey Replication (NCS-R). JAMA. 2003;289(23):3095-3105. doi:10.1001/ jama.289.23.3095

2. Abas MA, Phillips C, Carter J, Walter J, Banerjee S, Levy R. Culturally sensitive validation of screening questionnaires for depression in older African-Caribbean people living in south London. $\mathrm{Br}$ $J$ Psychiatry. 1998;173:249-254.

3. Lee Y, Yang MJ, Lai TJ, Chiu NM, Chau TT. Development of the Taiwanese Depression Questionnaire. Chang Gung Med J. 2000;23 (11):688-694.

4. Lee Y, Lin PY, Hsu ST, Cing-Chi Y, Yang LC, Wen JK. Comparing the use of the Taiwanese depression questionnaire and beck depression inventory for screening depression in patients with chronic pain. Chang Gung Med J. 2008;31(4):369-377.

5. Lee Y, Wu YS, Chien CY, Fang FM, Hung CF. Use of the Hospital Anxiety and Depression Scale and the Taiwanese Depression Questionnaire for screening depression in head and neck cancer patients in Taiwan. Neuropsychiatr Dis Treat. 2016;12:2649-2657.

6. Wang LJ, Chen CK, Hsu SC, Lee SY, Wang CS, Yeh WY. Active job, healthy job? Occupational stress and depression among hospital physicians in Taiwan. Ind Health. 2011;49(2):173-184. 
7. Steffens DC, Skoog I, Norton MC, Hart AD, Tschanz JT, Plassman BL, Wyse BW, Welsh-Bohmer KA, Breitner JC. Prevalence of depression and its treatment in an elderly population: the Cache County study. Arch Gen Psychiatry. 2000;57(6):601-607.

8. Park M, Unutzer J. Geriatric depression in primary care. Psychiatr Clin North Am. 2011;34(2):469-487, ix-x.

9. Morimoto SS, Alexopoulos GS. Cognitive deficits in geriatric depression: clinical correlates and implications for current and future treatment. Psychiatr Clin North Am. 2013;36(4):517-531.

10. Grayson L, Thomas A. A systematic review comparing clinical features in early age at onset and late age at onset late-life depression. J Affect Disord. 2013;150(2):161-170. doi:10.1016/j.jad.2013.03.021

11. Balsamo M, Cataldi F, Carlucci L, Padulo C, Fairfield B. Assessment of late-life depression via self-report measures: a review. Clin Interv Aging. 2018;13:2021-2044.

12. Blazer DG. Depression in late life: review and commentary. J Gerontol a Biol Sci Med Sci. 2003;58(3):249-265.

13. Caine ED. Pseudodementia: current Concepts and Future Directions. JAMA Psychiatry. 1981;38(12):1359-1364.

14. Hill NL, Mogle J, Wion R, et al. Subjective Cognitive Impairment and Affective Symptoms: a Systematic Review. Gerontologist. 2016;56(6):e109-e127.

15. Srisurapanont M, Mok YM, Yang YK, et al. Cognitive complaints and predictors of perceived cognitive dysfunction in adults with major depressive disorder: findings from the Cognitive Dysfunction in Asians with Depression (CogDAD) study. J Affect Disord. 2018;232:237-242.

16. Thomas AJ, Gallagher P, Robinson LJ, et al. A comparison of neurocognitive impairment in younger and older adults with major depression. Psychol Med. 2009;39(5):725-733.

17. Yesavage JA, Brink TL, Rose TL, et al. Development and validation of a geriatric depression screening scale: a preliminary report. J Psychiatr Res. 1982;17(1):37-49.

18. Morimoto SS, Kanellopoulos D, Alexopoulos GS. Cognitive impairment in depressed older adults: implications for prognosis and treatment. Psychiatr Ann. 2014;44(3):138-142.

19. Massai P, Colalelli F, Sansoni J, et al. Reliability and validity of the geriatric depression scale in Italian subjects with Parkinson's disease. Parkinsons Dis. 2018;2018:7347859.

20. Galeoto G, Sansoni J, Scuccimarri M, et al. Evaluation of the Italian Version of the Geriatric Depression Scale. Depress Res Treat. 2018;2018:1797536.

21. Liu C-Y, Lu C-H, Yu S, Yang -Y-Y. Correlations between scores on Chinese versions of long and short forms of the geriatric depression scale among elderly Chinese. Psychol Rep. 1998;82(1):211-214.

22. Liao YC, Yeh TL, Yang YK, et al. Reliability and Validation of the Taiwan Geriatric Depression Scale. Taiwanese J Psychiatry. 2004;18(1):30-41.

23. Yang YH, Galvin JE, Morris JC, Lai CL, Chou MC, Liu CK. Application of $\mathrm{AD} 8$ questionnaire to screen very mild dementia in Taiwanese. Am J Alzheimers Dis Other Demen. 2011;26(2):134-138.

24. Galvin JE, Roe CM, Xiong C, Morris JC. Validity and reliability of the AD8 informant interview in dementia. Neurology. 2006;67 (11):1942-1948.
25. Galvin JE, Roe CM, Powlishta KK, et al. The AD8: a brief informant interview to detect dementia. Neurology. 2005;65(4):559-564.

26. Wei YC, Huang LY, Chen CK, et al. Subjective cognitive decline in the community is affected at multiple aspects of mental health and life quality: a cross-sectional study of the community medicine of keelung chang gung memorial hospital. Dement Geriatr Cogn Dis Extra. 2019;9(1):152-162.

27. Lecrubier Y, Sheehan D, Weiller E, et al. The Mini International Neuropsychiatric Interview (MINI). A short diagnostic structured interview: reliability and validity according to the CIDI. Eur Psychiatry. 1997;12(5):224-231.

28. Sheehan DV, Lecrubier Y, Sheehan KH, et al. The Mini-International Neuropsychiatric Interview (M.I.N.I): the development and validation of a structured diagnostic psychiatric interview for DSM-IV and ICD-10. J Clin Psychiatry. 1998;59(Suppl 20):22-33.

29. Shyu YI, Yip PK. Factor structure and explanatory variables of the Mini-Mental State Examination (MMSE) for elderly persons in Taiwan. J Formos Med Assoc. 2001;100(10):676-683.

30. Buysse DJ, Reynolds CF 3rd, Monk TH, Berman SR, Kupfer DJ. The Pittsburgh sleep quality index: a new instrument for psychiatric practice and research. Psychiatry Res. 1989;28(2):193-213.

31. Lu JF, Tseng HM, Tsai YJ. Assessment of health-related quality of life in Taiwan (I): development and Psychometric Testing of SF-36 Taiwan Version. Taiwan J Public Health. 2003;22:501-511.

32. Ferrari AJ, Charlson FJ, Norman RE, et al. Burden of depressive disorders by country, sex, age, and year: findings from the global burden of disease study 2010. PLoS Med. 2013;10(11):e1001547.

33. Aseltine RH Jr, Kessler RC. Marital disruption and depression in a community sample. J Health Soc Behav. 1993;34(3):237-251.

34. Sasson I, Umberson DJ. Widowhood and Depression: new Light on Gender Differences, Selection, and Psychological Adjustment. J Gerontol B Psychol Sci Soc Sci. 2013;69B(1:135-145.

35. Ramlall S, Chipps J, Bhigjee AI, Pillay BJ. The sensitivity and specificity of subjective memory complaints and the subjective memory rating scale, deterioration cognitive observee, mini-mental state examination, six-item screener and clock drawing test in dementia screening. Dement Geriatr Cogn Disord. 2013;36(1-2):119-135.

36. Niemann U, Brueggemann P, Boecking B, Mazurek B, Spiliopoulou M. Development and internal validation of a depression severity prediction model for tinnitus patients based on questionnaire responses and socio-demographics. Sci Rep. 2020;10 (1):4664.

37. Choi B, Shim G, Jeong B, Jo S. Data-driven analysis using multiple self-report questionnaires to identify college students at high risk of depressive disorder. Sci Rep. 2020;10(1):7867.

38. Mitchell AJ, Bird V, Rizzo M, Meader N. Diagnostic validity and added value of the geriatric depression scale for depression in primary care: a meta-analysis of GDS30 and GDS15. J Affect Disord. 2010;125(1):10-17.

39. Chin WC, Liu CY, Lee CP, Chu CL. Validation of five short versions of the geriatric depression scale in the elder population in Taiwan. Taiwanese J Psychiatry. 2014;28(3):156-163.
Neuropsychiatric Disease and Treatment

\section{Publish your work in this journal}

Neuropsychiatric Disease and Treatment is an international, peerreviewed journal of clinical therapeutics and pharmacology focusing on concise rapid reporting of clinical or pre-clinical studies on a range of neuropsychiatric and neurological disorders. This journal is indexed on PubMed Central, the 'PsycINFO' database and CAS, an is the official journal of The International Neuropsychiatric Association (INA). The manuscript management system is completely online and includes a very quick and fair peer-review system, which is all easy to use. Visit http://www.dovepress.com/testimonials.php to read real quotes from published authors. 